\title{
Selective Fluidization of Synaptosomal Plasma Membrane Vesicles by $17 \beta$-Estradiol
}

\author{
Sae A Lee, Yong Jin Park, Il Ho Jang and Jung Sook Kang ${ }^{\dagger}$ \\ Department of Oral Biochemistry and Molecular Biology, School of Dentistry, \\ Pusan National University, Yangsan 50612, Korea
}

\begin{abstract}
Estrogens are effective neuroprotectants in vivo and in vitro. To obtain a better insight into the molecular mechanisms of action of neuroprotection by $17 \beta$-estradiol (E2), we examined the differential effects of E2 on the fluidity of synaptosomal plasma membrane vesicles (SPMV) isolated from rat cerebral cortex. Intramolecular excimerization of 1,3-di(1-pyrenyl)propane (Py-3-Py) was used to investigate the effects of E2 on the bulk and annular lateral diffusion of the SPMV. In addition, we examined the effects of E2 on the rotational diffusion of individual leaflet of SPMV exploiting selective quenching of outer monolayer 1,6-diphenyl-1,3,5-hexatriene (DPH) fluorescence by trinitrophenyl groups. The Förster distance $R_{0}$ value for the tryptophan-Py-3-Py donor-acceptor pair was $26.9 \AA$. E2 increased the lateral mobility of both bulk and annular lipids in SPMV in a dose-dependent manner, but a larger effect on bulk lipids was observed. Although E2 decreased the anisotropy of DPH in SPMV, E2 had a greater fluidizing effect on the outer leaflet compared to the inner leaflet. These results suggest that E2 selectively fluidizes the more fluid regions within SPMV. It is highly probable that E2 mostly fluidizes the bulk lipids, away from either annular lipids or lipid rafts, in the outer leaflet of SPMV. This selective fluidization may be one of the nongenomic mechanisms of neuroprotection by E2.
\end{abstract}

Key Words: $17 \beta$-Estradiol, Selective fluidization, Nongenomic, Neuroprotection, Förster distance

\section{INTRODUCTION}

Estrogens have shown beneficial effects against a variety of neuronal insults both in vivo and in vitro. Consistent observations of potent neuroprotection by estrogens made this ovarian steroid a prime candidate for protection of a variety of cell types during chronic neurodegenerative diseases, e.g., Alzheimer's disease and Parkinson's disease, as well as acute brain cell-compromising conditions, such as stroke and brain trauma (Brann et al., 2007; McCullough and Hurn, 2003). Although the major conducted clinical trials found detrimental effects of estrogen replacement therapy in postmenopausal women including increased stroke risk and dementia (Liu and Yang, 2013), estrogens are better studied for their neuroprotective effects than any other class of compounds. Most studies on neuroprotection of estrogen have been initially focused on the classical genomic pathways involving the binding of estrogen to the cognate nuclear estrogen receptors (ER), $\mathrm{ER}_{\alpha}$ and $\mathrm{ER}_{\beta}$ (Hall et al., 2001). There is a growing body of evidence that neuroprotection of estrogen includes nongenomic actions through binding to $\mathrm{ER}_{\alpha}, \mathrm{ER}_{\beta}$, or G-protein coupled ER, GPR30/GPER1 in the plasma membrane or through functioning independently

* Received: November 2, 2016 / Revised: December 23, 2016 / Accepted: December 26, 2016

$\dagger$ Corresponding author: Jung Sook Kang. Department of Oral Biochemistry and Molecular Biology, School of Dentistry, Pusan National University, Yangsan 50612, Korea

Tel: +82-51-510-8226, Fax:+82-51-510-8228, e-mail: jsokang@pusan.ac.kr

(C) The Korean Society for Biomedical Laboratory Sciences. All rights reserved.

(c) This is an Open Access article distributed under the terms of the Creative Commons Attribution Non-Commercial License (http://creativecommons.org/licenses/by-nc/3.0/) which permits unrestricted non-commercial use, distribution, and reproduction in any medium, provided the original work is properly cited. 
from ER (Alexander et al., 2016; Manavathi and Kumar, 2006). The genomic action is generally detectable in hours to days whereas nongenomic responses of cells occur in seconds to minutes. Rapid signaling by estrogens has been widely reported in numerous hormone target cells of the brain (Raz et al., 2008).

A large, diverse collection of physiological agonists produces the alterations in membrane fluidity as well as their specific ligand-receptor interaction. Although a few studies were performed about the membrane actions of $17 \beta$-estradiol (E2), conflicting results were reported depending on the types of membranes and the probe location. E2 increased (Dicko et al., 1999; Kumar et al., 2011; Tsuda et al., 2001; Whiting et al., 1995, 2000), decreased (Schwartz et al., 1996), or did not change (Golden et al., 1999; Shivaji and Jagannadham, 1992) membrane fluidity. All fluorescence spectroscopic studies about the effects of E2 on the membrane fluidity were performed using either 1,6-diphenyl1,3,5-hexatriene (DPH) or 1-[4-(trimethyammonio)-phenyl]6-phenylhexa-1,3,5-triene, both of which reflect the bulk rotational diffusion of membrane bilayers. Given the structural complexity of the bilayer lipids, it is prudent to characterize the fluidity of a given membrane in terms of intrinsically different modes of motion. The excimer fluorescence and fluorescence polarization reflect the lateral and rotational diffusion, respectively, of the fluorophores.

The aims of this research are threefold: (i) to investigate the effects of E2 on the bulk and annular lateral diffusion of synaptosomal plasma membrane vesicles (SPMV) isolated from rat cerebral cortex using intramolecular excimerization of 1,3-di(1-pyrenyl)propane (Py-3-Py); (ii) to examine the effects of E2 on the rotational diffusion of individual leaflet of SPMV exploiting selective quenching of outer leaflet DPH fluorescence by 2,4,6-trinitrobenzenesulfonic acid (TNBS); and (iii) to calculate the Förster distance of the fluorescence resonance energy transfer (FRET) from SPMV tryptophan to Py-3-Py. E2 increased both the lateral and the rotational mobility of SPMV, and this fluidizing effect was selective within SPMV. E2 preferentially increased the lateral mobility of bulk lipids than annular lipids. In view of rotational mobility, E2 selectively fluidized the outer leaflet than the inner leaflet.

\section{MATERIALS AND METHODS}

\section{Materials}

E2, TNBS, Ficoll, bovine serum albumin (BSA), and buffers were purchased from Sigma Chemical Co. (St. Louis, MO, USA); and DPH and Py-3-Py from Invitrogen (Carlsbad, CA, USA). All other chemicals were of the highest quality available, and water was deionized with a Milli-Q system.

\section{Animals}

We used the cerebral cortex of 12-month old SpragueDawley female rats. Animal experiments were performed according to the guidelines of the Korean Food and Drug Administration. All procedures were conducted in accordance with the ethical standards formulated by the Pusan National University Animal Care and Use Committee.

\section{Preparation of synaptosomes}

Synaptosomes were isolated from the cerebral cortex of adult (12-month old) Sprague-Dawley female rats by the method of Yun and Kang (1990) with several modifications. All steps were carried out at $4^{\circ} \mathrm{C}$. Rat cerebral cortex was homogenized in $0.32 \mathrm{M}$ sucrose $+0.25 \mathrm{mM}$ ethylenediaminetetraacetic acid (EDTA) (pH 7.4) and centrifuged at $580 \times \mathrm{g}$ for $10 \mathrm{~min}$ in a JA-25.50 rotor (Beckman Avanti $\mathrm{J}-25$ centrifuge). The supernatant was then spun at 12,000 $\times \mathrm{g}$ for $20 \mathrm{~min}$. The resulting pellet was suspended in 0.32 M sucrose $+10 \mathrm{mM}$ HEPES ( $\mathrm{pH} 7.4$ ) and layered over 7.5 and $14 \%$ Ficoll in $0.32 \mathrm{M}$ sucrose $+0.25 \mathrm{mM}$ EDTA +10 $\mathrm{mM}$ HEPES ( $\mathrm{pH}$ 7.4). The gradients were centrifuged at $87,000 \times \mathrm{g}$ for $30 \mathrm{~min}$ in a SW28 rotor (Beckman L80M ultracentrifuge). The synaptosomal band at the $7.5 \sim 14 \%$ interface was collected, diluted with $0.32 \mathrm{M}$ sucrose +10 $\mathrm{mM}$ HEPES and centrifuged at 17,200 $\times \mathrm{g}$ for $20 \mathrm{~min}$.

TNBS Labelling reactions and plasma membrane isolation

TNBS labeling reactions and preparation of plasma membrane vesicles were performed by the procedure of Yun and Kang (1990). Synaptosomal pellet was gently suspended in 
$2 \mathrm{mM}$ TNBS + buffer A or buffer A alone. Buffer A was composed of $30 \mathrm{mM} \mathrm{NaCl}, 120 \mathrm{mM} \mathrm{NaHCO}, 11 \mathrm{mM}$ glucose, $2 \% \mathrm{BSA}, \mathrm{pH} 8.5$. To assure complete exposure of all outer monolayers to TNBS, the pellet was passed slowly through a glass Dounce homogenizer (3 up and down strokes). All treatments were carried out at $4{ }^{\circ} \mathrm{C}$ for $40 \mathrm{~min}$. The TNBS labeling reaction was terminated by adding $1 \%$ BSA in phosphate-buffered saline (PBS). The suspension was immediately centrifuged at $17,200 \times \mathrm{g}$ for $15 \mathrm{~min}$. The resulting pellet was suspended in $0.32 \mathrm{M}$ sucrose $+10 \mathrm{mM}$ HEPES (pH 7.4), layered over 7.5 and 14\% Ficoll in $0.32 \mathrm{M}$ sucrose $+0.25 \mathrm{mM}$ EDTA + $10 \mathrm{mM}$ HEPES (pH 7.4), and centrifuged at $87,000 \times \mathrm{g}$ for $30 \mathrm{~min}$. The $7.5 \sim 14 \%$ interface was removed, diluted with $0.32 \mathrm{M}$ sucrose $+10 \mathrm{mM}$ HEPES, and centrifuged at $17,200 \times \mathrm{g}$ for $20 \mathrm{~min}$. The synaptosomal pellet was resuspended in $5 \mathrm{mM}$ Tris- $\mathrm{HCl}$ ( $\mathrm{pH} 8.5$ ) and allowed to lyse. The suspension was vortexed every $20 \mathrm{~min}$ for $1 \mathrm{hr}$ and centrifuged at $41,000 \times \mathrm{g}$ for 20 min. The pellet was resuspended in cold distilled water, layered over $0.75 \mathrm{M}$ sucrose $+0.25 \mathrm{mM}$ EDTA + $10 \mathrm{mM}$ HEPES (pH 7.4), and centrifuged at 41,600 $\times \mathrm{g}$ for $30 \mathrm{~min}$. The interface was removed and centrifuged at 43,500 $\times \mathrm{g}$ for $20 \mathrm{~min}$. The pellet was resuspended in PBS at a protein concentration of $5 \sim 10 \mathrm{mg} / \mathrm{ml}$. The purity was evaluated by morphological and enzymatic standards. Protein was determined by the method of Lowry et al. (1951) with BSA as a standard.

\section{Fluorescence measurements}

Aliquots of SPMV were suspended in PBS (pH 7.4) at a protein concentration of $50 \mu \mathrm{g} / \mathrm{ml}$. Py-3-Py stock solution was prepared in chloroform, and stock solution of DPH was made in dimethylformamide. The Py-3-Py concentration was $0.5 \mu \mathrm{M}$ while the concentration of $\mathrm{DPH}$ was $1 \mu \mathrm{M}$. The concentrations of Py-3-Py and DPH were determined using molar extinction coefficients of 80,000 and $88,000 \mathrm{M}^{-1} \mathrm{~cm}^{-1}$ at 344 and $350 \mathrm{~nm}$, respectively. The incorporation of Py3-Py was started by adding aliquots of a stock solution to membrane suspension. The mixture was incubated at $4{ }^{\circ} \mathrm{C}$ for $18 \mathrm{hr}$ under gentle stirring. For incorporation of DPH, aliquots of DPH stock solution were added to the membrane suspension, and the mixture was incubated at $37^{\circ} \mathrm{C}$ for 30 min in the dark.

In order to determine the effect of E2 on membrane fluidity, appropriate concentrations of the stock solution of E2 in dimethylsulfoxide was added to the labeled membrane suspension, and the mixture was incubated at $37^{\circ} \mathrm{C}$ for $1 \mathrm{hr}$. For bulk lateral diffusion, the excitation wavelength of Py-3-Py was $330 \mathrm{~nm}$. Py-3-Py was excited through FRET from tryptophan on SPMV proteins $(286 \mathrm{~nm})$ to determine annular lateral diffusion. The excimer to monomer fluorescence intensity ratio (I'/I) value of Py-3-Py was calculated from the 480 to $379 \mathrm{~nm}$ signal ratio. The excitation wavelength of DPH was $360 \mathrm{~nm}$ while emission was observed at $430 \mathrm{~nm}$. Fluorescence measurements were carried out at $37^{\circ} \mathrm{C}$ using a Cary Eclipse fluorescence spectrophotometer (Varian Inc., Palo Alto, CA, USA).

The DPH anisotropy is calculated by

$$
r=\frac{I_{V V}-G I_{V H}}{I_{V V}+2 G I_{V H}}
$$

where the intensity of the components of the fluorescence that were parallel $\left(I_{V V}\right)$ and perpendicular $\left(I_{V H}\right)$ to the direction of the vertically polarized excitation light was determined by measuring the emitted light through polarizers oriented vertically and horizontally. The grating correction factor $\mathrm{G}$ is given by the ratio of the fluorescence intensities of the vertical $\left(I_{H V}\right)$ to horizontal $\left(I_{H H}\right)$ components when the exciting light is polarized in the horizontal direction.

\section{Calculation of Förster distance}

The Förster distance $R_{0}$ is the distance at which FRET is $50 \%$ efficient and given by

$$
R_{0}=\left(J(\lambda) \kappa^{2} Q_{D} n^{-4}\right)^{1 / 6} \times 9.78 \times 10^{3} \AA
$$

where $J(\lambda)$ is the spectral overlap integral of donor emission and acceptor absorption, $k^{2}$ is the orientation factor for dipoledipole interaction, $Q_{D}$ is quantum yield of the donor in the absence of acceptor, and $\mathrm{n}$ is the refractive index of the medium. The overlap integral $J(\lambda)$ expresses the degree of spectral overlap between the donor emission and the acceptor absorption: 


$$
J(\lambda)=\int_{0}^{\infty} F_{D}(\lambda) \varepsilon_{A}(\lambda) \lambda^{4} d \lambda
$$

where $F_{D}(\lambda)$ is the corrected fluorescence intensity of the donor in the wavelength range $\lambda$ to $\lambda+\Delta \lambda$, with the total intensity (area under the curve) normalized to unity, and $\varepsilon_{A}(\lambda)$ is the extinction coefficient of the acceptor at $\lambda$, which is typically in the unit of $\mathrm{M}^{-1} \mathrm{~cm}^{-1}$.

\section{Determination of individual leaflet anisotropy in SPMV: Selective quenching of DPH by trinitrophenyl groups}

This experimental determination of individual leaflet anisotropy in SPMV is based on a method previously established for LM plasma membranes (Sweet et al., 1987) and synaptic plasma membranes (Schoeder et al., 1988). The individual leaflet anisotropy was calculated by

$$
r=\frac{F_{i}}{F} r_{i}+\frac{F-F_{i}}{F} r_{o}
$$

where $F$ and $F_{i}$ are fluorescence of DPH obtained for SPMV isolated from synaptosomes incubated with buffer $\mathrm{A}$ and buffer A plus TNBS at $4{ }^{\circ} \mathrm{C}$, respectively. The anisotropies $r$ (bulk anisotropy) and $r_{i}$ (inner leaflet anisotropy) were determined for DPH in SPMV obtained from synaptosomes incubated with buffer A and buffer A plus TNBS at $4{ }^{\circ} \mathrm{C}$, respectively. Equation was then solved for $r_{o}$ (outer leaflet anisotropy).

\section{Statistical analysis}

The data are presented as mean \pm SEM. Student's $t$-test was performed to determine the significant difference. Values were considered statistically significant when $P$ value was $<0.05$. The statistical analyses were performed using IBM SPSS Statistics 23 (SPSS Inc., Chicago, IL, USA).

\section{RESULTS}

\section{Effects of E2 on the bulk and the annular lateral diffusion of SPMV}

We evaluated the effect of E2 on lateral diffusion of SPMV using intramolecular excimerization of Py-3-Py. Py-

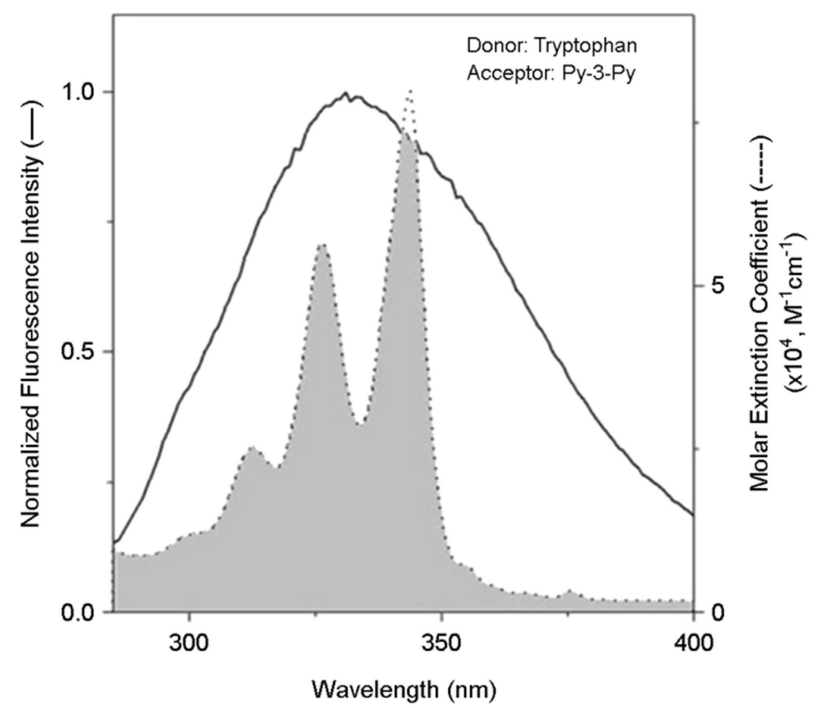

Fig. 1. Emission spectrum (-) of tryptophan in synaptosomal plasma membrane vesicles isolated from rat cerebral cortex and absorption spectrum $(\cdots)$ of Py-3-Py in methanol.

3-Py was excited either at its own excitation wavelength $(330 \mathrm{~nm})$ or through FRET at the excitation wavelength of tryptophan $(286 \mathrm{~nm})$. FRET is transfer of the excited state energy from an initially excited donor to an acceptor. The rate of energy transfer depends upon the extent of spectral overlap of the emission spectrum of the donor with the absorption spectrum of the acceptor, the quantum yield of the donor, the relative orientation of the donor and acceptor transition dipoles, and the distance between the donor and acceptor molecules (Lakowicz, 2006). Because of the simple operation under homogeneous conditions, it has been used as a power tool for investigating a number of molecular interactions. Fig. 1 shows the absorption and emission spectra of the tryptophan-Py-3-Py donor-acceptor pair. As can be seen, there is a significant overlap of the emission spectrum of the donor tryptophan with the absorption spectrum of the acceptor Py-3-py. The spectral overlap integral $(J(\lambda))$ was used to calculate the Förster distance $R_{0}$ according to Eq. (2), and the result is summarized in Table 1 . The $R_{0}$ value for the tryptophan-Py-3-Py donor-acceptor pair was $26.9 \AA$, which was comparable to the values for tryptophan-pyrene pair (Dobretsov et al., 1982; Tahara et al., 1992). In the calculation of $R_{0}$, the value of $k^{2}$ was $2 / 3$, and that of the 
Table 1. Donor quantum yield $\left(Q_{D}\right)$, spectral overlap integral $(J(\lambda))$ and Förster distance $\left(R_{0}\right)$ of tryptophan-Py-3-Py donoracceptor pair

\begin{tabular}{ccc}
\hline \hline$Q_{D}{ }^{\mathrm{a})}$ & $\begin{array}{c}J(\lambda)^{\mathrm{b})} \\
\left(\times 10^{-14}, \mathrm{M}^{-1} \mathrm{~cm}^{3}\right)\end{array}$ & $\begin{array}{l}R_{0}{ }^{\mathrm{b}} \\
(\AA)\end{array}$ \\
\hline 0.13 & 2.554 & 26.9 \\
\hline
\end{tabular}

a) From Chen (1967).

${ }^{b)} R_{0}$ and $J(\lambda)$ were calculated according to Eqs. (2) and (3), respectively.

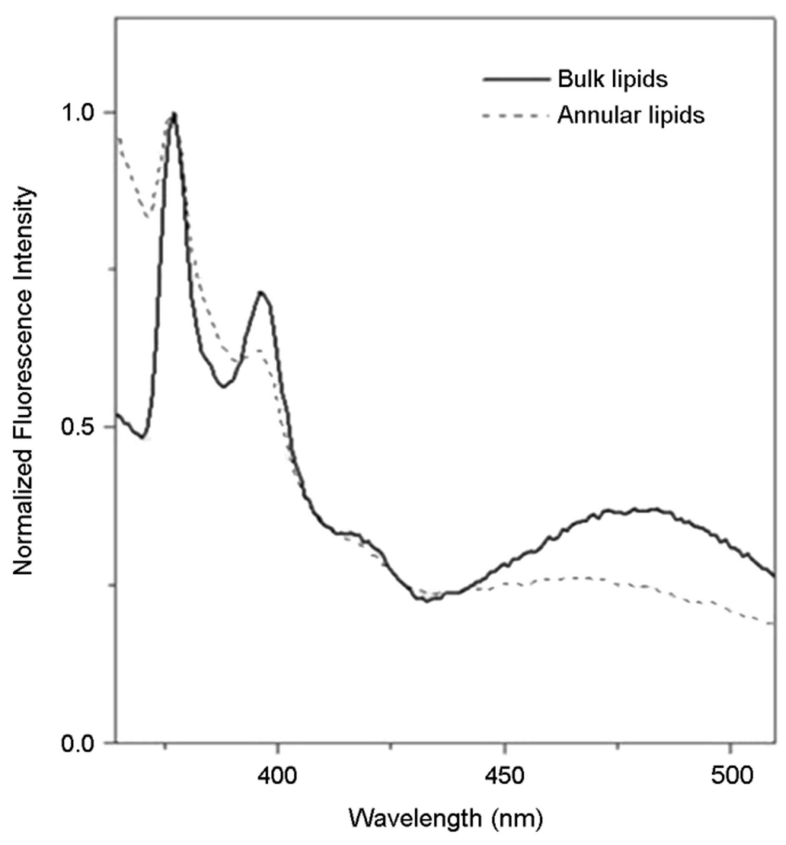

Fig. 2. Emission spectra of Py-3-Py in the synaptosomal plasma membrane vesicles isolated from rat cerebral cortex. Fluorescence measurements were performed at $37^{\circ} \mathrm{C}(\mathrm{pH} 7.4)$. The excitation wavelengths of Py-3-Py were 330 and $286 \mathrm{~nm}$ for bulk and annular lipids, respectively.

refractive index ( $n$ ) was taken as 1.5 (Lakowicz, 2006).

The normalized fluorescence spectra of Py-3-Py in bulk and annular lipids of SPMV are shown in Fig. 2. In SPMV, bulk lipids were more fluid than annular lipids. At $37^{\circ} \mathrm{C}$, the I'/I values of Py-3-Py in bulk and annular lipids of SPMV were $0.417 \pm 0.009$ and $0.280 \pm 0.008$, respectively. Fig. 3 shows the effect of E2 on the bulk and annular lateral diffusion of SPMV. E2 dose-dependently increased the I'/I ratio of the probe molecule, i.e., the lateral mobility of both bulk and annular lipids in SPMV. However, we could easily

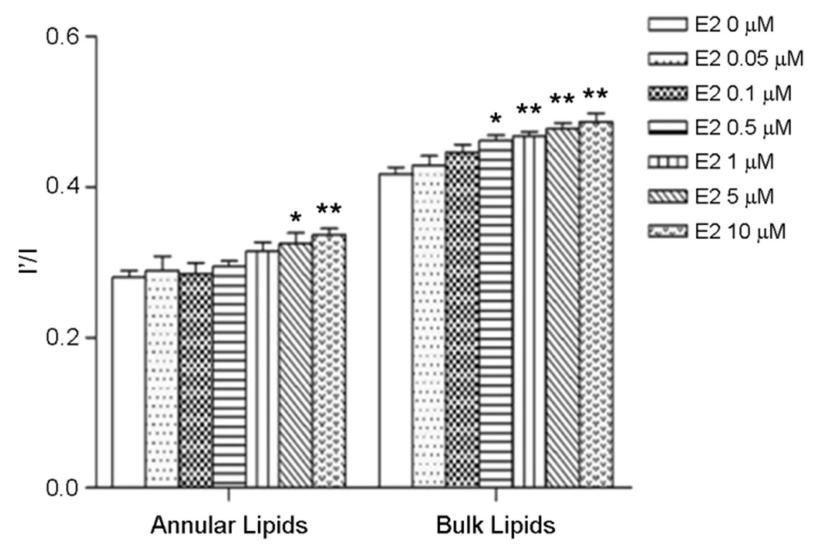

Fig. 3. Effects of E2 on the bulk and the annular lateral diffusion of the synaptosomal plasma membrane vesicles isolated from rat cerebral cortex. The excitation wavelengths of Py-3-Py were 330 and $286 \mathrm{~nm}$ for bulk and annular lipids, respectively. The excimer to monomer fluorescence intensity ratio (I'/I) of Py-3-Py was calculated from the 480 to $379 \mathrm{~nm}$ signal ratio. Fluorescence measurements were performed at $37^{\circ} \mathrm{C}(\mathrm{pH} \mathrm{7.4)}$. Each point represents the mean \pm SEM of 5 determinations. ${ }^{*} P<0.05,{ }^{*} P<0.01$ vs. control (no E2 treatment).

see that the increases in bulk lipids were larger than those in annular lipids within SPMV.

\section{Differential effects of $\mathbf{E} 2$ on the transbilayer rotational diffusion in SPMV}

Selective quenching of DPH fluorescence by trinitrophenyl groups was utilized to examine the transbilayer effects of E2 on the rotational mobility of SPMV. The validity of this method has been well demonstrated in a variety of the plasma membrane vesicles (Schroeder et al., 1988; Sweet et al., 1987). The $R_{0}$ value for DPH-trinitrophenyl donoracceptor pair is $16.6 \AA$ (Radda, 1975), and the thickness of biological membranes is usually between $40 \sim 80 \AA$. So, the anisotropy values for TNBS-treated SPMV are those for inner leaflet, and the anisotropy values for outer leaflet were calculated using Eq. 4.

The effects of increasing concentrations of E2 on the anisotropy of DPH incorporated into SPMV are shown in Fig. 4. E2 dose-dependently decreased the anisotropy of $\mathrm{DPH}$, indicating the increase in rotational mobility of bulk SPMV. The increase in both the lateral and rotational mobility in SPMV indicates the bulk lipid fluidization. However, 


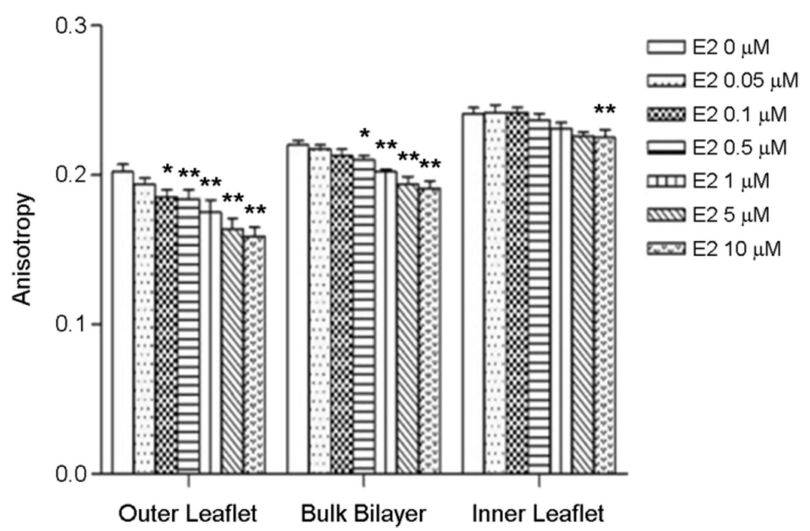

Fig. 4. Selective effects of E2 on transbilayer anisotropy $(r)$ of DPH in the synaptosomal plasma membrane vesicles isolated from rat cerebral cortex. Synaptosomes were treated $\pm 2 \mathrm{mM} 2,4,6-$ trinitrobenzenesulfonic acid (TNBS), $\mathrm{pH} 8.5$, at $4^{\circ} \mathrm{C}$ for $40 \mathrm{~min}$, and the plasma membrane vesicles were isolated. DPH was incorporated, and fluorescence measurements were performed at $37^{\circ} \mathrm{C}$. Untreated and TNBS-treated membranes represent the bulk bilayer and the inner leaflet, respectively. The bulk and inner leaflet anisotropy values were obtained by Eq. (1), and the outer leaflet anisotropy was calculated using Eq. (4). Each point represents the mean \pm SEM of 5 determinations. ${ }^{*} P<0.05,{ }^{* *} P<0.01$ vs. control (no E2 treatment).

E2 showed a greater decreasing effect on the outer leaflet anisotropy as compared to the inner leaflet anisotropy. We observed a significant decrease in the outer leaflet anisotropy of DPH even at $100 \mathrm{nM}$. In the inner leaflet, however, a significant decrease was observed only at $10 \mu \mathrm{M}$. Our data presented herein have shown that the increase in bulk bilayer rotational diffusion is mainly due to an increase in the outer leaflet rotational diffusion of SPMV.

\section{DISCUSSION}

The main point of the present study is that E2 exhibits a selective rather than nonselective fluidizing effect within SPMV. E2 fluidized bulk lipids than annular lipids from the point of view of lateral mobility. From the viewpoint of rotational mobility, E2 selectively fluidized outer leaflet than inner leaflet. Even at a concentration in the nanomolar range $(100 \mathrm{nM})$, the increase in rotational mobility of the outer leaflet of SPMV was observed. These results indicate a selective fluidizing effect of $\mathrm{E} 2$ on the more fluid regions within SPMV, which can partly explain the inconsistent effects of E2 on membrane fluidity.

One explanation for the selective fluidizing effect of E2 may be the asymmetric distribution of cholesterol within transbilayer domains of SPMV. Cholesterol is the key molecule which determines membrane fluidity. The inner monolayer of SPMV was enriched in sterol, with lower sterol levels in the outer monolayer. Whiting et al. (1995) reported that the steroid hormone effect on membrane fluidity was less pronounced in both liposomes and native membranes containing cholesterol. It has been proposed that cholesterol limits steroid hormones intercalating between lipid bilayers. It has been well established that membranes are heterogeneous, containing discrete detergent-resistant microdomains called lipid rafts that are enriched with cholesterol and sphingolipids (Simons and Ikonen, 1997). Many receptors including ER and signaling molecules are likely to be localized in lipid rafts and caveolae, a special type of lipid raft (Maselli et al., 2015). Lipid rafts play an important role in cellular signaling, functioning as physical platforms to concentrate and assemble the signal transduction machinery (Pralle et al., 2000). Lipid rafts have been shown to regulate signal transduction by activating or suppressing the phosphorylation cascades related to cell growth, survival, death, and other physiological processes (Patra, 2008). In view of the observation that E2 preferentially fluidized the more fluid regions within SPMV, it is not likely that E2 may induce significant fluidity changes in cholesterol-rich lipid rafts. In other words, it is probable that E2 may selectively fluidize the more fluid regions excluding lipid rafts even within the outer leaflet of SPMV. There has been emerging notion that, in order for a membrane to be functional, local rather than global fluidity plays a crucial role. Taken together, these results suggest that E2 is most likely to fluidize the bulk lipids, away from either annular lipids or lipid rafts, in the outer leaflet of SPMV. The selective fluidization of the more fluid regions within SPMV may be one of the nongenomic mechanisms of neuroprotection by E2.

Although the findings of this study cannot provide exact evidence for neuroprotection by E2, membrane fluidity can influence the dynamics of proteins and other biomolecules within the membrane, thereby affecting the functions of these molecules (Nicolson, 2014; Zhu et al., 2015). However, 
the present results might still underestimate the effect of E2 on the fluidity of specific membrane domains because only the bulk and annular lateral mobility and the bulk and transbilayer rotational mobility of SPMV were determined. In addition, further experimentation is required to elucidate how the selective fluidization of the bulk lipids in the outer leaflet affects the ER embedded in lipid rafts of neuronal membranes.

\section{Acknowledgements}

This work was supported by a 2-Year Research Grant of Pusan National University.

\section{Conflict of interest}

None.

\section{REFERENCES}

Alexander A, Irving AJ, Harvey J. Emerging roles for the novel estrogen-sensing receptor GPER1 in the CNS. Neuropharmacology. 2016. 113: 652-660.

Brann DW, Dhandapani K, Wakade C, Mahesh VB, Khan MM. Neurotrophic and neuroprotective actions of estrogen: basic mechanisms and clinical implications. Steroids. 2007. 72: 381 -405 .

Chen RF. Fluorescence quantum yields of tryptophan and tyrosine. Anal Lett. 1967. 1: 35-42.

Dicko A, Morissette M, Ameur BS, Pézolet M, Paolo DT. Effect of estradiol and tamoxifen on brain membranes: investigation by infrared and fluorescence spectroscopy. Brain Res Bull. 1999. 49: 401-405.

Dobretsov GE, Spirin MM, Chekrygin OV, Karmansky IM, Dmitriev VM, Vladimirov Yu A. A fluorescence study of apolipoprotein localization in relation to lipids in serum low density lipoproteins. Biochim Biophys Acta. 1982. 710: 172 -180 .

Golden GA, Mason RP, Tulenko TN, Zubenko GS, Rubin RT. Rapid and opposite effects of cortisol and estradiol on human erythrocyte $\mathrm{Na}^{+}, \mathrm{K}^{+}$-ATPase activity: relationship to steroid intercalation into the cell membrane. Life Sci. 1999. 65: 1247 -1255 .

Hall JM, Couse JF, Korach KF. The multifaceted mechanisms of estradiol and estrogen receptor signaling. J Biol Chem. 2001. 276: 36869-36872.
Kumar P, Taha A, Kale RK, McLean P, Baquer NZ. Protective effects of $17 \beta$ estradiol on altered age related neuronal parameters in female rat brain. Neurosci Lett. 2011. 502: 56-60.

Lakowicz JR. Principles of fluorescence spectroscopy. 2006.

Liu R, Yang SH. Window of opportunity: estrogen as a treatment for ischemic stroke. Brain Res. 2013. 1514: 83-90.

Lowry OH, Rosebrough NR., Farr AL, Randall RJ. Protein measurement with the Folin phenol reagent. J Biol Chem. 1951. 193 265-275.

Manavathi B, Kumar R. Steering estrogen signals from the plasma membrane to the nucleus: two sides of the coin. J Cell Physiol 2006. 207: 594-604.

Maselli A, Pierdominici M, Vitale C, Ortona E. Membrane lipid rafts and estrogenic signaling: a functional role in the modulation of cell homeostasis. Apoptosis. 2015. 20: 671-678.

McCullough LD, Hurn PD. Estrogen and ischemic neuroprotection an integrated view. Trends Endocrinol Metab. 2003. 14: 228 -235 .

Nicolson GL. The fluid-mosaic model of membrane structure: still relevant to understanding the structure, function and dynamics of biological membranes after more than 40 years. Biochim Biophys Acta. 2014. 1838: 1451-1466.

Patra SK. Dissecting lipid raft facilitated cell signaling pathways in cancer. Biochim Biophys Acta. 2008. 1785: 182-206.

Pralle A, Keller P, Florin EL, Simons K, Hrber JK. Sphingolipidcholesterol rafts diffuse as small entities in the plasma membrane of mammalian cells. J Cell Biol. 2000. 148: 997-1008.

Radda GK. Fluorescence probes in membrane studies in methods in membrane biology, biophysical approaches. Plenum Press 1975. 4: 97-188.

Raz L, Khan MM, Mahesh VB, Vadlamudi RK, Brann DW. Rapid estrogen signaling in the brain. Neurosignals. 2008. 16: 140 -153 .

Schroeder F, Morrison WJ, Gorka C, Wood WG. Transbilayer effects of ethanol on fluidity of brain membrane leaflets. Biochim Biophys Acta. 1988. 946: 85-94.

Schwartz Z, Gates PA, Nasatzky E, Sylvia VL, Mendez J, Dean DD, Boyan BD. Effect of 17ß-estradiol on chondrocyte membrane fluidity and phospholipid metabolism is membranespecific, sex-specific, and cell maturation-dependent. Biochim Biophys Acta. 1996. 1282: 1-10.

Shivaji S, Jagannadham MV. Steroid-induced perturbations of membranes and its relevance to sperm acrosome reaction Biochim Biophys Acta. 1992. 1108: 99-109.

Simons K, Ikonen E. Functions rafts in cell membranes. Nature. 
1997. 387: 569-572.

Sweet WD, Wood WG, Schroeder F. Charged anesthetics selectively alter plasma membrane order. Biochemistry. 1987. 26: 2828-2835.

Tahara Y, Murata M, Ohnishi S, Fujiyoshi Y, Kikuchi M, Yamamoto Y. Functional signal peptide reduces bilayer thickness of phosphatidylcholine liposomes. Biochemistry. 1992. 31: 8747-8754.

Tsuda K, Kinoshita Y, Kimura K, Nishio I, Masuyama Y. Electron paramagnetic resonance investigation on modulatory effect of $17 \beta$-estradiol on membrane fluidity of erythrocytes in postmenopausal women. Arterioscler Thromb Vasc Biol. 2001. 21: 1306-1312.
Whiting KP, Brain PF, Restall CJ. Steroid hormone induced effects on membrane fluidity. Biochem Soc Trans. 1995. 23: 438.

Whiting KP, Restall CJ, Brain PF. Steroid hormone-induced effects on membrane fluidity and their potential roles in non-genomic mechanisms. Life Sci. 2000. 67: 743-757.

Yun I, Kang JS. The general lipid composition and aminophospholipid asymmetry of synaptosomal plasma membrane vesicles isolated from bovine cerebral cortex. Mol Cells. 1990. 1: 15-20.

Zhu D, Bungart BL, Yang X, Zhumadilov Z, Lee JC, Askarova S. Role of membrane biophysics in Alzheimer's-related cell pathways. Front Neurosci. 2015. 9: 186. 